\section{Plan Nacional de Salud Mental en Chile: 10 años de experiencia}

\author{
Alberto Minoletti ${ }^{1}$ \\ y Alessandra Zaccaria ${ }^{2}$
}

Palabras clave: programas nacionales de salud, planes y programas de salud, salud mental, Chile.

\footnotetext{
Ministerio de Salud, Departamento de Salud Mental, Santiago, Chile. La correspondencia debe dirigirse a Alberto Minoletti, Departamento de Salud Mental, Ministerio de Salud, Mac-Iver 459, Piso 8, Santiago, Chile. Correo electrónico: aminolet@minsal.cl

2 Servicio de Psiquiatría, Hospital del Salvador, Santiago, Chile.
}

La solución de los problemas de salud mental constituye indiscutiblemente una tarea cada vez más importante de la salud pública en el mundo. Se calcula que 450 millones de personas sufren actualmente de trastornos mentales (1). El impacto de las enfermedades mentales, tanto para la persona afectada como para sus familiares y la sociedad en general, es muy alto, no solo en términos emocionales, sino también financieros. Se calcula que el gasto ocasionado por estos trastornos puede equivaler a $3-4 \%$ del producto nacional bruto de los países desarrollados $(2,3)$.

En Chile, la prevalencia en la vida de los principales trastornos mentales es de $36 \%$ y los más frecuentes son la agorafobia $(11,1 \%)$, la depresión mayor $(9,0 \%)$, la distimia $(8,0 \%)$ y la dependencia del alcohol (6,4\%). En general, estas afecciones explican $15 \%$ del total de años de vida saludable perdidos por enfermedad (4-8). Esta situación requiere necesariamente de una conducta activa por parte del Estado, lo que en Chile se tradujo en la aplicación de estrategias de salud pública agrupadas en dos planes nacionales de salud mental y psiquiatría puestos en marcha por el Ministerio de Salud en los años 1993 y 2000 (9-11). Con el primer plan se introdujeron prácticas innovadoras que sirvieron de base para formular el segundo plan, que estableció un modelo más elaborado de red de atención y de programas de actividades que abarcaban los problemas prioritarios.

El plan aplicado en el año 2000 reflejaba las principales líneas de acción propuestas por la Organización Mundial de la Salud (OMS) en su Informe sobre la salud en el mundo en el año 2001 (1). Entre esas líneas de acción se encuentran el desarrollo de servicios comunitarios de salud mental, la integración de la atención de salud mental en los servicios de salud generales y la creación de vínculos intersectoriales. Estos programas tienen un enfoque biopsicosocial y dan prioridad a las acciones preventivas y de intervención temprana dirigidas a mejorar continuamente la calidad de la atención, con la participación activa de los usuarios, sus familiares y organizaciones locales.

En el presente artículo se describe el modelo de atención de salud mental y psiquiatría aplicado en el sistema público de salud de Chile, se analizan las principales dificultades encontradas y los logros obtenidos en los 10 años que lleva ese plan en funcionamiento y se hacen recomendaciones para perfeccionarlo. 


\section{EL SISTEMA DE SALUD EN CHILE}

En Chile, el sistema de salud es mixto, aunque predomina el sector estatal (figura 1), que es el que atiende a la población más pobre, de mayor edad y con mayor carga de enfermedades $(12,13)$. El organismo coordinador del Sistema Nacional de Servicios de Salud (SNSS) es el Ministerio de Salud, que cumple funciones rectoras y normativas y tiene a su cargo 28 servicios de salud descentralizados territorialmente. Su organismo de financiamiento es el Fondo Nacional de Salud (FONASA), que funciona como un proveedor de seguro público de salud. En respuesta al grave deterioro en que se encontraba el sector de la salud a fines de la década de 1980 debido a la reducción del presupuesto estatal de salud en el período de 1973 a 1989, los recursos asignados a este sector han aumentado sostenidamente a fin de mejorar la cobertura y la calidad de los servicios. El aumento promedio del gasto público en salud en el período 1989-2000 fue de 8,1\%, superior al promedio del sector social, que fue de 5,4\% (14-16). Durante la década de 1990, el gasto en salud estuvo concentrado en los sectores más pobres de la población con el objetivo de contribuir a mejorar la calidad de la vida de ese segmento poblacional. La estrategia general aplicada por el Ministerio de Salud se ha reflejado también en la asignación de más recursos para la atención de los problemas de salud mental de la población de Chile.

Los cambios en los indicadores de salud, en la cantidad de recursos y en el número de actividades realizadas en los últimos años han sido grandes y positivos y han ubicado a Chile a la vanguardia de la Región por sus indicadores de salud, con logros reconocidos internacionalmente (17-20). Como consecuencia de la transición demográfica, la esperanza de vida al nacer de los chilenos es actual-

FIGURA 1. Estructura del sistema de salud de Chile, 2004

\begin{tabular}{lc}
\multicolumn{2}{c}{ SISTEMA DE SALUD DE CHILE } \\
SECTOR PRIVADO & SECTOR PÚBLICO \\
- 18 aseguradoras, Institucio- & - Un gran ente de \\
nes de Salud Previsional & financiamiento, el Fondo \\
(ISAPRES), cubren a & Nacional de Salud \\
2720 060 beneficiarios & (FONASA), atiende a \\
(17,6\% de la población). & 10580 090 beneficiarios \\
- Red de prestadores de & (68,3\% de la población). \\
servicios, la mayor parte & - 28 servicios de salud \\
privados, en los principales & articulados en una compleja \\
centros urbanos. & red nacional de hospitales \\
- La Superintendencia de & públicos (383) y centros \\
ISAPRES (SISP) regula & de atención primaria \\
estas aseguradoras. & municipal (526). \\
Fuente: Ministerio de Salud e Instituto Nacional de Estadísticas, Chile. \\
a El Ministerio de Salud regula ambos sectores.
\end{tabular}

Fuente: Ministerio de Salud e Instituto Nacional de Estadísticas, Chile. ${ }^{a}$ El Ministerio de Salud regula ambos sectores. mente de 76,7 años y la tasa de mortalidad general es de 5,74 por 1000 habitantes. Sin embargo, se debe destacar que el suicidio es la séptima causa de muerte en hombres, con una tasa de 16,8 por 100000 habitantes de ese sexo (21).

Dado el cambio en el perfil epidemiológico de la población chilena, se hace cada vez más importante mejorar la calidad de la vida y reducir la prevalencia de las enfermedades que producen discapacidad, en primer lugar los trastornos mentales y del comportamiento. Una gran parte del absentismo y de la disminución del rendimiento laboral, así como del aumento en el número de accidentes, enfermedades físicas y muertes prematuras, se relaciona con los trastornos mentales.

\section{Plan Nacional de Salud Mental y Psiquiatría}

El Plan Nacional de Salud Mental y Psiquiatría recoge y sistematiza la experiencia de trabajo de muchas personas que - en su condición de usuarios, familiares de los usuarios, técnicos y profesionales de la salud- hicieron un valioso aporte a la reflexión y al análisis colectivo que permitió llegar a este plan. En él se señalan los objetivos y las estrategias que se deben seguir para orientar los recursos del Estado hacia acciones que mejoren el bienestar y la salud mental de los chilenos. El Plan tiene como propósito contribuir a que las personas, las familias y las comunidades alcancen y mantengan la mayor capacidad posible para relacionarse entre sí y con el medio ambiente, de modo que promuevan el bienestar subjetivo, el desarrollo y el uso óptimo de sus potencialidades psicológicas, cognoscitivas, afectivas y relacionales, y el logro de sus metas individuales y colectivas, en concordancia con la justicia y el bien común (10).

El Plan Nacional de Salud Mental y Psiquiatría hace hincapié en el enfoque comunitario de los problemas de salud mental. Además, se basa en intervenciones fundamentadas en los últimos avances científicos y toma en cuenta criterios económicos, tales como la eficacia en función del costo. En términos estratégicos, este plan establece que se deben crear servicios de salud mental y psiquiatría en red -dentro del sector de la salud y junto con otros sectores- en los que participen el usuario y sus familiares y en los que las organizaciones locales desempeñen un papel destacado en el desarrollo de los servicios $(22,23)$.

\section{MODELO DE INTERVENCIÓN}

Desde la década de 1950, diversos factores — como el desarrollo de nuevos psicofármacos y el 
FIGURA 2. Esquema de la red de salud mental y psiquiatría. Chile, 2004

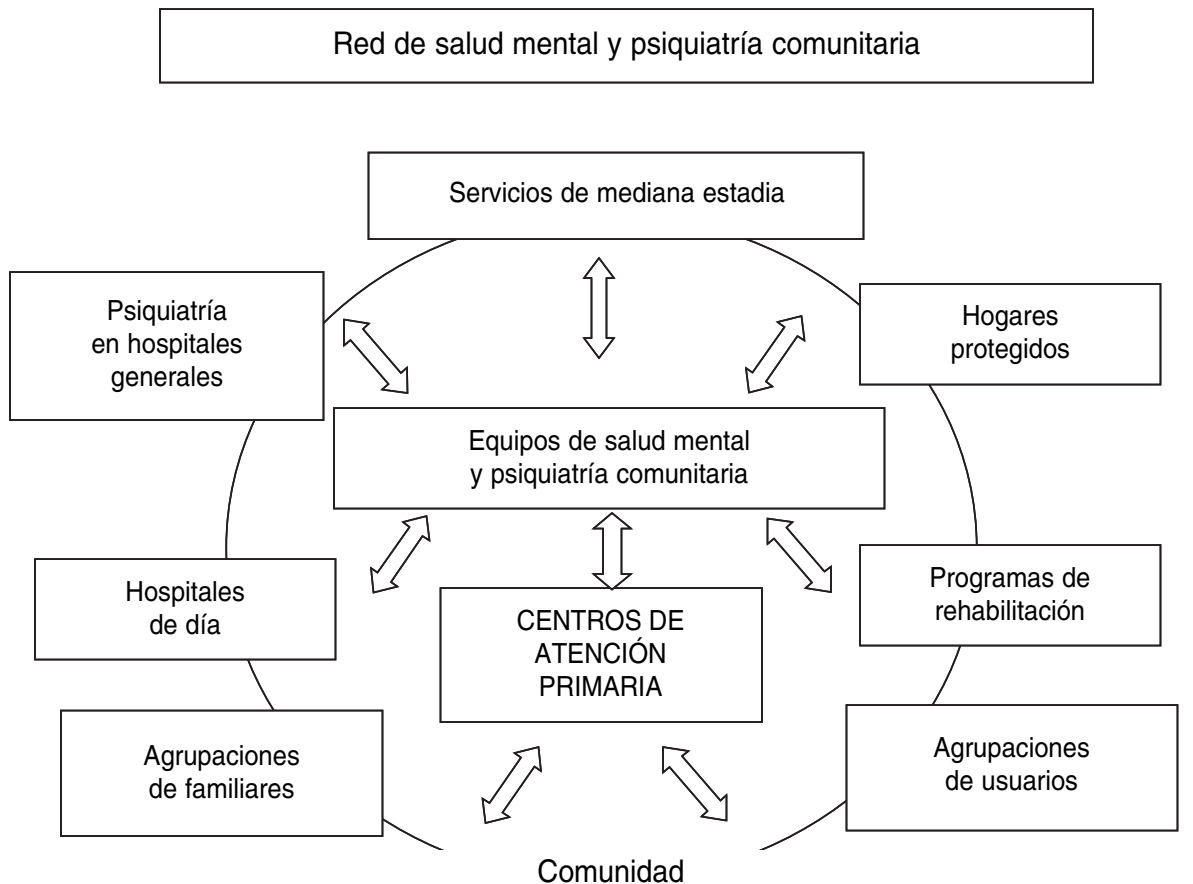

Fuente: Ministerio de Salud, Chile.

establecimiento de un enfoque nuevo respecto de los trastornos psiquiátricos graves basado en criterios psicológicos, sociológicos y antropológicoshan contribuido a la evolución de los modelos de intervención para trastornos psiquiátricos, desde el nivel hospitalario hasta el comunitario.

Gracias a las políticas aprobadas por el Estado para la salud mental, en Chile se ha venido adoptando paulatinamente el modelo comunitario en los últimos 10 años, considerado por muchos investigadores como el mejor modelo para la atención de trastornos psiquiátricos, tanto desde el punto de vista ético como terapéutico. Los resultados obtenidos mediante la aplicación de la psiquiatría comunitaria han demostrado que los factores biológicos explican solo parcialmente la etiopatogenia de los trastornos psiquiátricos, ya que algunos factores psicosociales condicionan su aparición y duración mediante una compleja interacción entre la persona, su entorno social inmediato y sus antecedentes (24).

El desarrollo del modelo de atención comunitario permite que los recursos asistenciales disponibles, ya sean públicos o privados, se apliquen a los trastornos de salud mental y a las enfermedades psiquiátricas de la población de una determinada área geográfica en forma coordinada e integrada, dando prioridad a las estrategias comu- nitarias y participativas, según las realidades y necesidades de cada género y grupo sociocultural. Según este modelo, el eje del apoyo organizativo es el equipo de salud mental y psiquiatría comunitaria (figura 2).

La red de salud mental y psiquiatría funciona por territorios y tiene bajo su responsabilidad la atención de los usuarios del sistema de seguridad pública de salud, las emergencias y los servicios convenidos para los afiliados de los sistemas privados de seguros de salud, así como las acciones de salud pública dirigidas a toda la población. Este modelo cumple con las recomendaciones de la OMS para la organización de los servicios de salud mental y es parte del Modelo General de Atención en Salud que se aplica actualmente en todo el país como parte de la reforma del sector de la salud. Además, este modelo general reconoce que la salud es un derecho humano básico y busca lograr una mayor equidad en el acceso a los servicios, una mayor calidad en la atención sanitaria, y la desconcentración y descentralización de los servicios de salud y de la toma de decisiones. Esta forma de abordar los problemas de salud mental favorece especialmente las acciones de promoción y prevención y el fortalecimiento de la atención primaria, con una buena capacidad resolutiva enfocada en las personas, las familias y las comunidades. 


\section{Estrategias}

Intersectorialidad en salud mental. El Plan Nacional de Salud Mental y Psiquiatría define acciones específicas que el sector de la salud debe coordinar con otros sectores del gobierno y de la sociedad civil, y es un primer paso hacia la elaboración de una política y de un plan intersectorial de salud mental en Chile. Para los próximos años se han definido cuatro ámbitos de acción: las escuelas, los centros de trabajo, los barrios y comunas, y las personas en conflicto con la justicia.

Programa de Salud Mental. Este programa tiene el propósito de orientar la programación de los recursos y las prestaciones del sistema. Prevé la detección, el tratamiento y la rehabilitación de las personas con trastornos mentales, así como actividades de promoción y de prevención, según el nivel de resolución, el equipo o establecimiento en que se realiza la acción y el contexto general de la red de servicios del territorio de que se trate (25).

Prioridades programáticas. Las prioridades programáticas se definieron tomando en cuenta algunos indicadores epidemiológicos - como la prevalencia-, el impacto social, los tratamientos y las intervenciones más eficaces en función del costo, y la disponibilidad de recursos humanos capacitados en salud mental y psiquiatría. Para la primera etapa de aplicación del plan (2000-2010) se dio prioridad a las siguientes acciones:

- promoción de la salud mental y de la prevención de los riesgos

- atención a los niños y adolescentes en edad escolar con trastornos de atención e hipercinéticos

- atención a las personas con trastornos mentales asociados con la violencia (maltrato infantil, violencia intrafamiliar y represión política durante el período 1973-1990)

- atención a las personas con depresión

- atención a las personas con trastornos psiquiátricos graves, especialmente con esquizofrenia

- atención a las personas con la enfermedad de Alzheimer y otras formas de demencia

- atención a las personas con dependencia del alcohol y las drogas.

Red de servicios de salud mental y psiquiatría. La creación y el fortalecimiento de la red de servicios de salud mental y psiquiatría comunitaria contribuyen a afianzar el modelo de atención propuesto en el Plan Nacional. El objetivo de esta red es facilitar el acceso a los servicios y a las acciones sanitarias en instalaciones más cercanas a su residencia. Durante el año 1999, el Ministerio de Salud aprobó la Norma
Técnica No. 35, que reguló la organización de una red de servicios de salud mental y psiquiatría y orientó las funciones, las relaciones y las actividades de los distintos componentes de esa red. Esta red debe garantizar la diversidad y frecuencia de las prestaciones requeridas por los pacientes y sus familiares y la continuidad de los cuidados, tomando en cuenta las necesidades de las diferentes poblaciones, las etapas del ciclo de vida y las particularidades del género (26). El desarrollo de los recursos y de los establecimientos de esta red se presenta en los cuadros 1 y 2 , respectivamente.

Estructura organizativa. La estructura organizativa de los servicios de salud mental sigue el mismo enfoque del Sistema de Salud de Chile. La Unidad de Salud Mental del Ministerio de Salud es el órgano rector y normativo, mientras que en los 28 servicios de salud del país funcionan unidades de salud mental encargadas de la gestión de los programas en sus respectivas áreas geográficas. Estas unidades están constituidas, en su mayoría, por profesionales de diversas disciplinas que trabajan a tiempo parcial, pero muy motivados y comprometidos con la salud mental de las personas y de la comunidad. Su función principal es garantizar la puesta en marcha y el funcionamiento de los programas locales, desarrollar y coordinar la red de salud mental y promover la relación con otros sectores y actores locales a fin de dar la mejor respuesta a las necesidades de la población en su área de acción.

Inversiones para la salud mental. La puesta en marcha del Plan Nacional de Salud Mental y Psiquiatría requiere de mayor financiamiento público con nuevos recursos y - solo de manera secundaria - la reorientación de los fondos existentes. El plan propone incrementar el presupuesto destinado a la salud mental entre 0,5 y 1,0\% anual a partir del presupuesto general de salud, de manera que el aumento alcance el 5\% de dicho presupuesto en 5-10 años. Esta propuesta se fundamenta en el impacto que tienen las inversiones en la promoción, la prevención, la detección y el tratamiento oportunos. Además, representan un gran ahorro debido a la reducción del número de tratamientos prolongados - de mayor costo- y de licencias médicas, servicios de rehabilitación y servicios de apoyo social (27).

Regulación. Con el objetivo de mejorar el nivel de salud y de garantizar altos estándares de calidad en la atención sanitaria (28), la Unidad de Salud Mental del Ministerio de Salud ha elaborado desde 1990 un grupo de regulaciones que establecen cómo organizar la atención de salud mental y psiquiatría. Además, se desarrollan sistemas para evaluar los estándares de calidad de los diversos estableci- 
CUADRO 1. Disponibilidad de recursos en la red de servicios de salud mental y psiquiatría del sistema público de salud. Chile, 1990-2003

\begin{tabular}{lrrrr}
\hline & \multicolumn{4}{c}{ Años } \\
\cline { 2 - 5 } \multicolumn{1}{c}{ Recurso } & 1990 & 1999 & 2001 & 2003 \\
\hline Psiquiatras & 224 & 412 & 436 & 498 \\
Psicólogos en atención primaria & 0 & 120 & 247 & 452 \\
Camas para estadías cortas $^{a}$ & 239 & 336 & 328 & 329 \\
$\quad$ en hospitales generales & 604 & 558 & 494 & 378 \\
$\quad$ en hospitales psiquiátricos & 0 & 0 & 74 & 96 \\
Camas para estadías medias $^{b}$ & 516 & 1689 & 1169 & 1029 \\
Camas para estadías largas $^{c}$ & 40 & 40 & 60 & 80 \\
Camas para psiquiatría forense $_{\text {Plazas en hospitales de día }}$ & 0 & 194 & 543 & 640 \\
Plazas en hogares protegidos & 0 & 200 & 530 & 783 \\
Plazas en centros de rehabilitación & 0 & 732 & 1588 & 1535 \\
\hline
\end{tabular}

Fuente: Departamento de Estadísticas e Información de Salud y Unidad de Salud Mental, Ministerio de Salud, Chile.

${ }^{a}$ Camas para estadías cortas: camas en hospitales generales o psiquiátricos que ofrezcan tratamientos especializados a base de un régimen de hospitalización completa por un tiempo máximo de de 60 días.

b Camas para estadías medianas: camas en hospitales psiquiátricos que cuenten con la debida acreditación del Ministerio de Salud para prestar atención a personas con enfermedades psiquiátrica o discapacidad psicosocial graves o resistentes, y que requieran tratamiento en régimen de hospitalización por un tiempo máximo de 12 meses.

c Camas para estadías largas: camas en hospitales generales o psiquiátricos, remanentes del antiguo modelo de atención para la custodia de por vida de personas con enfermedades psiquiátricas o discapacidad psicosocial graves o resistentes al tratamiento. Por instrucción del Ministerio de Salud se ha suspendido el ingreso de nuevas personas en esta modalidad desde el año 2001

mientos de salud mental y psiquiatría del sector público.

Agrupaciones de usuarios y familiares. Las organizaciones formales de usuarios de servicios de salud y de sus familiares son relativamente nuevas en Chile. A inicios de la década de 1990, con la recuperación democrática y los primeros esfuerzos por crear servicios modernos de salud mental, surgió con fuerza el movimiento de familiares de pacientes con trastornos mentales graves. En el Plan Nacional de Salud Mental y Psiquiatría del año 2000 se resalta explícitamente el papel y la utilidad de estas agrupaciones en todas las etapas del tratamiento y se promueve la interacción constructiva entre los profesionales y esas agrupaciones. Además, se expone la conveniencia de motivar y capacitar a personas con aptitudes de líder para que dirijan esos movimientos.

\section{Dificultades y falencias}

Resistencia natural al cambio. Como en todo proceso de transformaciones profundas, la puesta en marcha del Plan Nacional de Salud Mental y Psi-

CUADRO 2. Disponibilidad de establecimientos en la red de servicios de salud mental y psiquiatría. Chile, 1994-2003

\begin{tabular}{|c|c|c|c|c|c|c|c|}
\hline \multirow[b]{2}{*}{ Centros } & \multicolumn{6}{|c|}{ Año } & \multirow{2}{*}{$\begin{array}{c}\text { Incremento } \\
\text { entre } 1994 \text { y } 2003, \\
\%\end{array}$} \\
\hline & 1994 & 1996 & 1998 & 2000 & 2002 & 2003 & \\
\hline Equipos de salud mental y psiquiatría comunitaria & 0 & 0 & 4 & 8 & 39 & 42 & \\
\hline Centros de atención ambulatoria & 22 & 26 & 30 & 41 & 52 & 62 & 182 \\
\hline Hospitales de día & 3 & 5 & 6 & 10 & 31 & 36 & 1100 \\
\hline Centros diurnos & 9 & 16 & 17 & 19 & 22 & 24 & 167 \\
\hline Clubes de integración social & 0 & 3 & 5 & 9 & 43 & 46 & \\
\hline Hogares protegidos & 5 & 17 & 19 & 49 & 60 & 82 & 1540 \\
\hline
\end{tabular}

Fuente: Unidad de Salud Mental, Ministerio de Salud, Chile. 
quiatría ha despertado temores. Los trabajadores sanitarios con más años de experiencia en los servicios psiquiátricos tradicionales se sienten inseguros con el modelo comunitario y no ven claramente los beneficios del cambio, muchas veces por falta de conocimientos y de capacitación en el tema. Este fenómeno se ve agudizado por la insuficiencia de los planes de desarrollo de recursos humanos específicos para los servicios de salud mental y psiquiatría que permitan aplicar y dar continuidad a este modelo de atención.

Ampliación presupuestaria. En los últimos años, los recursos disponibles para el Plan Nacional de Salud Mental y Psiquiatría, asignados por los Ministerios de Salud y de Hacienda según las prioridades definidas para el presupuesto de la nación, han sido inferiores a lo propuesto en el Plan. Solo tres de las prioridades programáticas -la esquizofrenia, la depresión y la dependencia del alcohol y de las drogas- cuentan con financiamiento seguro por estar entre las 56 enfermedades del programa de Acceso Universal con Garantías Explícitas (AUGE).

Distribución de recursos. En Chile, la distribución geográfica de los recursos, tanto humanos como de infraestructura, muestra desigualdades e inequidades, con una marcada concentración de psiquiatras en los servicios de salud que cuentan con hospitales psiquiátricos (Aconcagua, Valparaíso, Metropolitano Sur y Metropolitano Norte) y en el de Iquique, como se observa en el cuadro 3 (29).

Sistema de registro e información de salud mental. El desarrollo del sistema de registro e información de salud mental como parte de las estadísticas de salud

CUADRO 3. Distribución de recursos humanos y físicos en los servicios secundarios de salud, según la región. Chile, 2002

\begin{tabular}{|c|c|c|c|c|c|}
\hline \multirow[b]{2}{*}{ Servicio de salud } & \multicolumn{3}{|c|}{ Horas semanales de psiquiatría } & \multicolumn{2}{|c|}{$\begin{array}{c}\text { Camas } \times 100000 \\
\text { beneficiarios del } \\
\text { seguro público }\end{array}$} \\
\hline & Contratadas & $\%$ & $\begin{array}{l}\text { Por } 100000 \\
\text { beneficiarios del } \\
\text { seguro público }\end{array}$ & $\begin{array}{c}\text { Estadía } \\
\text { corta }\end{array}$ & $\begin{array}{c}\text { Estadía } \\
\text { larga }\end{array}$ \\
\hline Arica $^{a}$ & 93,9 & 0,9 & 70,4 & 15,3 & 0,0 \\
\hline Iquique & 218,0 & 2,0 & 177,0 & 16,7 & 0,0 \\
\hline Antofagasta ${ }^{a}$ & 242,0 & 2,2 & 93,3 & 8,2 & 5,2 \\
\hline Atacama $^{\mathrm{a}}$ & 211,4 & 1,9 & 108,3 & 1,1 & 0,0 \\
\hline Coquimbo ${ }^{a}$ & 382,0 & 3,5 & 87,0 & 2,0 & 0,0 \\
\hline Valparaíso & 461,9 & 4,2 & 134,6 & 15,5 & 0,0 \\
\hline Viña $a^{a}$ & 484,0 & 4,4 & 79,2 & 0,0 & 0,0 \\
\hline Aconcagua & 267,0 & 2,4 & 147,8 & 23,5 & 191,5 \\
\hline M. ${ }^{b}$ Oriente $^{a}$ & 491,0 & 4,5 & 95,5 & 3,5 & 0,0 \\
\hline M. ${ }^{b}$ Central ${ }^{a}$ & 506,4 & 4,6 & 99,4 & 0,0 & 0,0 \\
\hline M..$^{b}$ Sur & 1070,7 & 9,8 & 144,3 & 9,6 & 26,7 \\
\hline M. ${ }^{b}$ Norte & 2426,1 & 22,1 & 538,8 & 54,7 & 27,0 \\
\hline M. $^{\mathrm{b}}$ Occidente ${ }^{\mathrm{a}}$ & 628,6 & 5,7 & 80,4 & 3,2 & 0,0 \\
\hline M. ${ }^{b}$ Sur Oriente ${ }^{a}$ & 445,1 & 4,1 & 58,7 & 1,4 & 0,0 \\
\hline O’Higgins $^{\mathrm{a}}$ & 429,1 & 3,9 & 74,1 & 0,0 & 0,0 \\
\hline Maule & 318,2 & 2,9 & 45,0 & 2,2 & 0,0 \\
\hline Nuble ${ }^{a}$ & 192,5 & 1,8 & 57,2 & 3,0 & 0,0 \\
\hline Concepción & 514,0 & 4,7 & 118,1 & 12,1 & 0,0 \\
\hline Talcahuano $^{\mathrm{a}}$ & 231,0 & 2,1 & 82,5 & 0,0 & 0,0 \\
\hline Bío-Bíoa & 55,0 & 0,5 & 18,0 & 0,0 & 0,0 \\
\hline Arauco $^{a}$ & 44,0 & 0,4 & 36,0 & 0,0 & 0,0 \\
\hline Araucania Norte & 66,0 & 0,6 & 40,7 & 2,6 & 0,0 \\
\hline Araucania Sura & 396,6 & 3,6 & 77,7 & 3,5 & 0,0 \\
\hline Valdivia $^{a}$ & 217,5 & 2,0 & 79,7 & 7,4 & 0,0 \\
\hline Osorno ${ }^{a}$ & 159,8 & 1,5 & 83,8 & 11,7 & 0,0 \\
\hline Llanchipal $^{\mathrm{a}}$ & 253,0 & 2,3 & 78,6 & 5,4 & 0,0 \\
\hline Aysen $^{a}$ & 41,3 & 0,4 & 58,2 & 0,0 & 0,0 \\
\hline Magallanes & 116,6 & 1,1 & 112,1 & 16,0 & 0,0 \\
\hline Total & 10962,7 & 100,0 & 105,0 & 6,8 & 6,4 \\
\hline
\end{tabular}


FIGURA 3. Porcentaje del presupuesto del sector público de salud destinado a la salud mental. Chile, 1990-2003

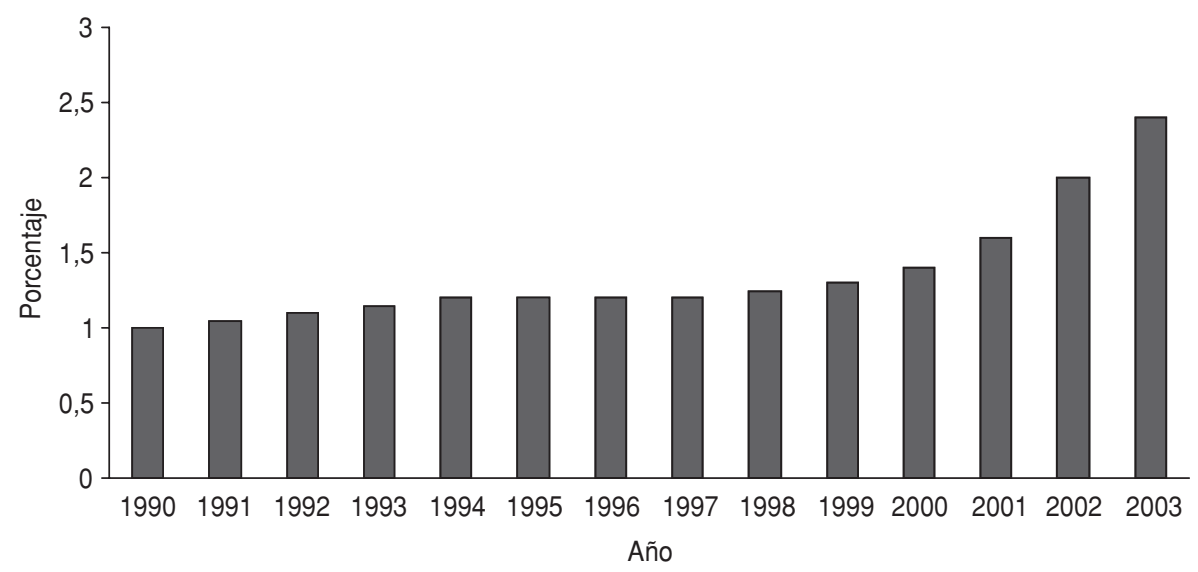

Fuente: Ministerio de Salud, Chile.

del sector público es aún incipiente. Hasta el año 2002 solo se registraba el número de prestaciones de salud mental, sin especificar la cantidad de personas beneficiadas y sus diagnósticos. A partir de ese año se comenzó a registrar la información con un mayor grado de desagregación y detalle, lo que ha permitido conocer el número de personas atendidas, de casos de abandono del tratamiento y de actividades de salud mental realizadas, tanto en el nivel primario como en el secundario, en los 28 servicios de salud del país. Dada su reciente aplicación y el poco desarrollo de las estructuras necesarias para su buen funcionamiento, la información es aún insuficiente.

Legislación. Hasta el momento no se cuenta con una ley de salud mental que garantice el desarrollo y la continuidad del nuevo modelo y que resguarde adecuadamente los derechos humanos de los pacientes $(30,31)$.

Salud mental infantil. A pesar de ser una de las prioridades programáticas del Plan Nacional de Salud Mental y Psiquiatría y uno de los objetivos sanitarios del Ministerio de Salud, el desarrollo de servicios de salud mental para niños y adolescentes está aún muy lejos de alcanzar la cobertura mínima definida en el Plan (32), ya que el Ministerio de Hacienda no ha destinado los recursos solicitados para esta área.

\section{RESULTADOS ALCANZADOS}

Los avances del sistema público de salud mental en Chile en los últimos 10 años son numerosos. A continuación se describen algunos de los más importantes.

\section{Aumento real y sostenido del presupuesto de salud mental}

Aunque los trastornos mentales y de la conducta constituyen $15 \%$ de la carga total de enfermedad, la inversión pública en salud mental en Chile era solo de $1 \%$ del presupuesto asignado al sector de la salud en 1990. A partir de entonces, la proporción del presupuesto de salud destinada a la salud mental ha aumentado sostenida y significativamente hasta llegar a 2,4\% del presupuesto asignado al sector de la salud en el año 2003 (figura 3). La distribución de los recursos financieros entre los distintos servicios ha cambiado, ya que antes de 1990 en los hospitales psiquiátricos se concentraba $74 \%$ del presupuesto de salud mental, mientras que en el año 2002, solo 43\% del presupuesto de salud mental correspondió a los hospitales psiquiátricos y $46 \%$ se dedicó a los centros comunitarios.

\section{Programas para trastornos mentales prioritarios}

De acuerdo con las definiciones del Plan Nacional, se han puesto en marcha en todo el país cuatro programas especializados para responder organizada y sistemáticamente a las necesidades de la población. Estos programas, prioritarios por su magnitud e impacto, han contado con normas técnicas y fondos específicos crecientes.

Programa para la detección, diagnóstico y tratamiento integral de la depresión. Este programa se inició en el año 2001 y en tres años se logró generalizar a todos los centros de atención primaria de salud de Chile, articulados con los centros de atención psi- 
FIGURA 4. Evolución del Programa Nacional de la Depresión en cuanto al número de personas atendidas y porcentaje derivado al nivel secundario de atención de salud. Chile, 2001-2004

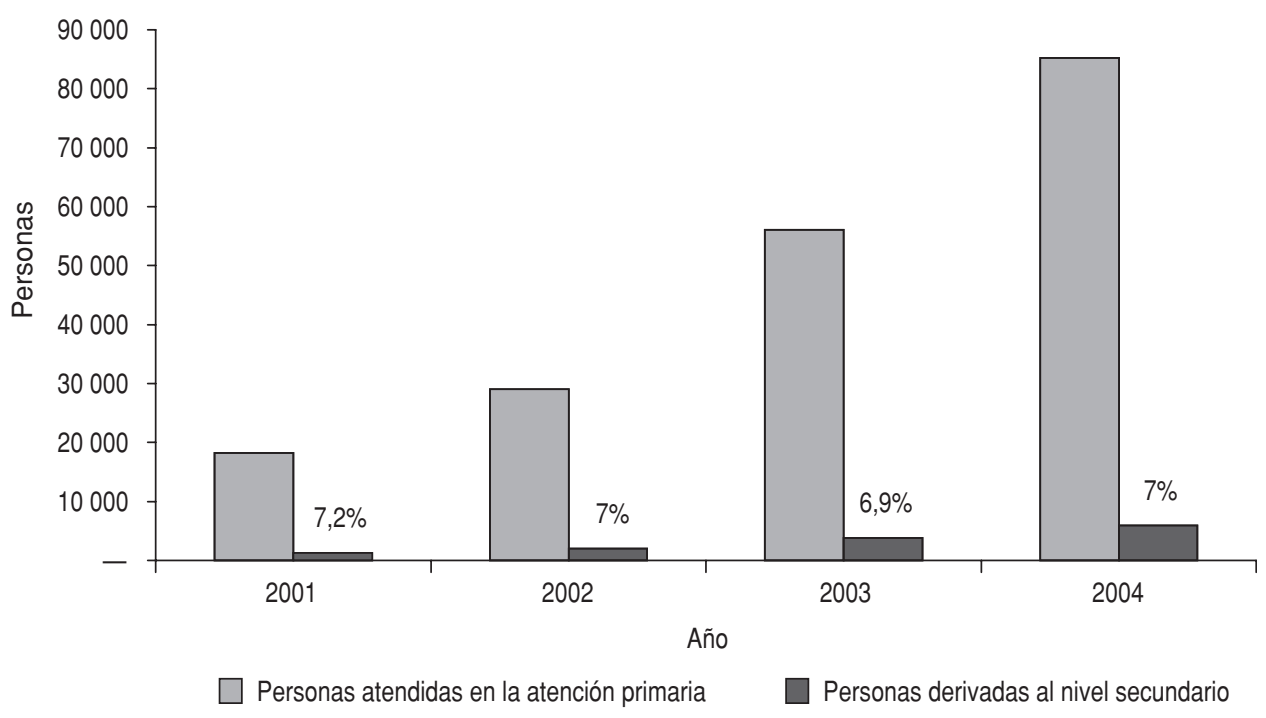

Fuente: Ministerio de Salud, Chile.

quiátrica. El programa presta atención especial al cuidado integral de los pacientes desde una perspectiva biopsicosocial, lo que ha ayudado a sistematizar y orientar el trabajo cotidiano de los equipos de salud mental y a mejorar la capacidad resolutiva de los equipos de atención primaria. El número de personas que ha accedido a este programa se ha triplicado en sus tres años de funcionamiento (figura 4) y en 2003 satisfacía 25\% de la demanda estimada del sistema público (4\% de los mayores de 20 años). De las personas tratadas ese año en el Programa Nacional, $92 \%$ eran mujeres con una edad promedio de 42 años. De ellas, $60 \%$ tenían antecedentes de algún episodio depresivo y $56 \%$ no contaban con apoyo de la red social. Una evaluación de impacto ha demostrado que en los beneficiarios de este programa se redujeron significativamente los síntomas de depresión, de angustia y de trastornos somáticos y en general manifestaron una mayor satisfacción con la vida y en la relación con su pareja (33).

Programa para el tratamiento de la esquizofrenia. El modelo de tratamiento propuesto por este programa se basa en estrategias comunitarias y en la atención ambulatoria de las personas con esquizofrenia mediante equipos multidisciplinarios, hospitales de día, unidades para la rehabilitación psicosocial y hogares protegidos y en la incorporación paulatina de antisicóticos atípicos — como la risperidona, la clozapina y la olanzapina- al tratamiento. Este programa se puso en marcha en el año 1999 para dar respuesta a la necesidad de tratar a las personas que acudían al sector público por presentar trastornos psiquiátricos graves o resistentes a los tratamientos habituales. En junio de 2004, alrededor de 12000 personas participaban en el programa y 7500 de ellas recibían fármacos de última generación. Además, a partir de abril de ese año se comenzó a aplicar un esquema de atención a personas que presentaban su primera manifestación de esquizofrenia, a fin de garantizar su acceso a una atención sanitaria oportuna y de calidad.

Programa para el tratamiento y la rehabilitación de personas con dependencia del alcohol y de drogas. Este programa se aplica en todos los servicios de salud del país desde el año 2001 y en él participan la red de atención primaria, los centros comunitarios de salud mental, los hospitales y las comunidades terapéuticas. Este programa ofrece tratamiento gratuito con cobertura limitada a los beneficiarios de FONASA en alguna de sus cuatro modalidades de tratamiento que, según las características, las necesidades y la complejidad del caso, son: plan de tratamiento como primera respuesta, plan de tratamiento ambulatorio básico, plan de tratamiento ambulatorio intensivo y plan de tratamiento residencial en comunidades terapéuticas. Se debe destacar que este programa lo lleva el Ministerio de Salud conjuntamente con el Consejo Nacional para el Control de Estupefacientes, dependiente del Ministerio del Interior, y el Ministerio de Justicia. Este programa constituye un excelente ejemplo de trabajo intersectorial (cuadro 4). 
CUADRO 4. Número de personas atendidas mediante el programa de tratamiento y rehabilitación para personas con problemas de dependencia del alcohol y de drogas, 2001-2003

\begin{tabular}{lrrrr}
\hline & \multicolumn{3}{c}{ Año } & \\
\cline { 2 - 4 } Modalidad de tratamiento & $2001^{\mathrm{a}}$ & 2002 & \multicolumn{1}{c}{2003} & Total \\
\hline Plan de primera respuesta & 1293 & 4495 & 6081 & 11869 \\
Plan ambulatorio básico & 725 & 733 & 3879 & 5337 \\
Plan ambulatorio intensivo & 275 & 272 & 1160 & 1707 \\
Plan residencial & 166 & 167 & 765 & 1098 \\
$\quad$ Total & 2459 & 5667 & 11885 & 20011 \\
\hline
\end{tabular}

Fuente: Fondo Nacional de Salud (FONASA), Consejo Nacional para el Control de Estupefacientes (CONACE) y Unidad de Salud Mental del Ministerio de Salud, Chile.

a Las cifras del año 2001 corresponden al período de septiembre a diciembre de ese año.

Programa de reparación integral de salud y derechos humanos (PRAIS). El Ministerio de Salud participa activamente en la aplicación de la Política Nacional de Reparación Social, elaborada por el primer gobierno democrático que sucedió a la dictadura militar que rigió los destinos de Chile entre 1973 y 1990. El PRAIS está orientado a la atención sanitaria integral física y mental, tanto de las personas afectadas por la represión política ejercida por el Estado en ese período como de sus familiares, ya sea de forma individual, familiar o sociocomunitaria. Una vez evaluada la magnitud del daño sufrido, se desarrollan intervenciones psicoterapéuticas en las que las propias personas afectadas participan activamente en su rehabilitación con la ayuda de organizaciones de usuarios, mediante actividades de autoayuda y de reinserción social. En septiembre de 1990 se conformó el primer equipo multiprofesional y un año después, el PRAIS se había extendido a 50\% de los servicios de salud. En la actualidad, todos los servicios de salud del país cuentan con personal capacitado para dar respuesta a las necesidades de los beneficiarios de este programa que en enero de 2004 contaba con más de 185000 participantes.

\section{Sistema Nacional de Psiquiatría Forense}

Este sistema, establecido en 1999, brinda atención oportuna - ya sea mediante tratamiento o programas de reinserción laboral y familiar - a las personas sobreseídas o absueltas judicialmente por padecer de alguna enfermedad mental. Las prestaciones de tratamiento y de rehabilitación a las personas que no representan un peligro para la comunidad se realizan mediante programas ambulatorios de psiquiatría forense en instalaciones de la red de centros de salud mental y psiquiatría y en hogares protegidos pertenecientes a los servicios de salud. El tratamiento de las personas que presentan algún grado de peligrosidad se realiza en la Unidad de Psiquiatría Forense de Alta Complejidad - que es una unidad de referencia nacional con 20 camas- o en alguna de las dos unidades de psiquiatría forense de mediana complejidad-que son unidades de referencia regional con un total de 60 camas.

Hasta 1998, la lista de espera para recibir tratamiento era de alrededor de 120 personas, sin embargo, la creación de estas unidades permitió reducir esta cifra a una cuarta parte. En la actualidad, alrededor de 70 personas sobreseídas ingresan anualmente en las unidades de psiquiatría forense, mientras otras 300 reciben tratamiento ambulatorio y 85 viven en hogares protegidos.

\section{Ampliación de la cobertura de la atención}

A partir de 1990 se ha ampliado la cobertura de atención de los principales trastornos psiquiátricos, especialmente con servicios que se prestan localmente. En un estudio realizado entre los años 1992 y 1999 se encontró que la cobertura de atención para personas con enfermedades mentales era de $38,5 \%$ (34). Esto se logró mediante la contratación de profesionales del área de la salud mental como parte de la aplicación del modelo comunitario, de forma que los problemas de salud mental de mayor demanda se podían atender en los consultorios de atención primaria de salud, ubicados en la zona de residencia de los usuarios. Esta estrategia permitió liberar recursos en los niveles de mayor complejidad y ayudó a revertir la tendencia histórica de concentrar la atención psiquiatría en los servicios especializados del nivel secundario. El aumento relativo de las actividades de salud mental fue mayor en el nivel primario que en el especializado (figura 5), aunque estos indicadores no reflejan toda la riqueza y el impacto de estas medidas. 
FIGURA 5. Número de prestaciones de salud mental del sector público, según el nivel de atención. Chile, 1993-2003

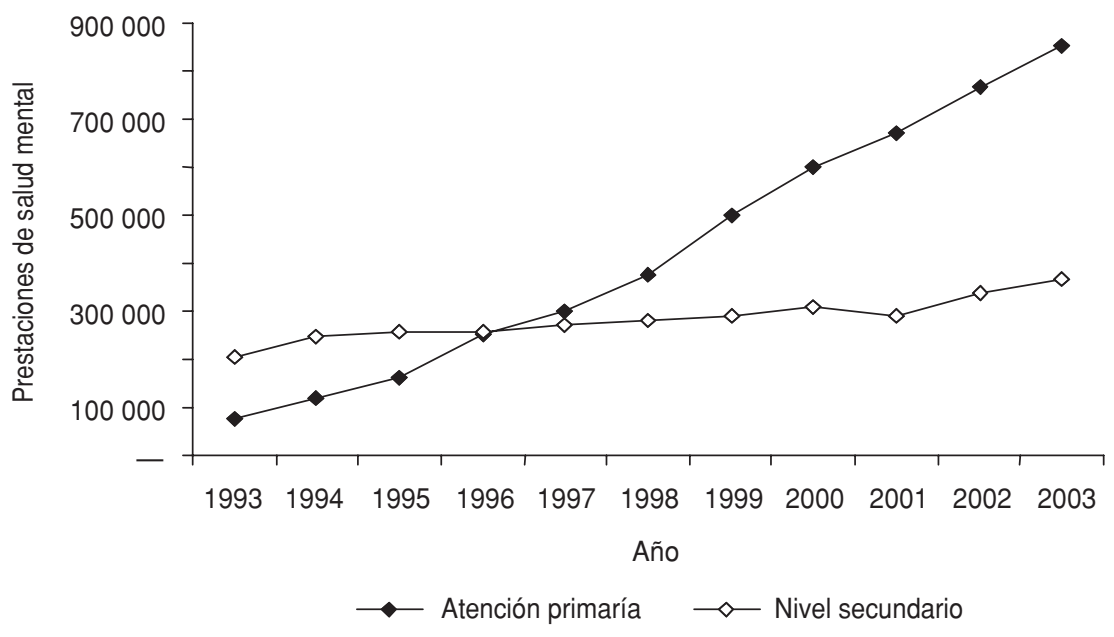

Fuente: Ministerio de Salud, Chile.

\section{Programa de mejoramiento continuo de la calidad}

En 2003 se inició la evaluación continua de las diferentes unidades de atención de la red de salud mental y psiquiatría. Ese año se evaluaron todos los hogares protegidos y hospitales de día, así como $30 \%$ de los equipos de salud mental y psiquiatría comunitaria. Esa evaluación abarcó diferentes aspectos de la calidad del servicio, entre ellas la competencia del sistema, su enfoque en los usuarios, el grado de satisfacción de las necesidades y el entorno laboral. Este programa permite evaluar el funcionamiento y la calidad de la atención que ofrecen los centros de la red de salud mental y psiquiatría.

\section{Satisfacción de los usuarios}

A cuatro años de la puesta en marcha de la segunda versión del Plan Nacional de Salud Mental y Psiquiatría se han publicado tres estudios que demuestran los avances logrados con la aplicación del modelo comunitario de atención en usuarios de los servicios públicos de salud (35-37). Según estos estudios, la aplicación del modelo comunitario está asociada con un mejor estado de salud mental de la población, lo que se expresa en una mayor puntuación en los indicadores de la calidad de la vida (figura 6), un mejor funcionamiento de las personas con enfermedad mental en la sociedad y una mayor satisfacción de los usuarios. En un estudio realizado recientemente (37), las personas con esquizofrenia que vivían en hogares protegidos mostraron un nivel de satisfacción con el servicio significativamente mayor que los que residían en servicios de larga estadía.

\section{Impacto en las políticas de salud}

A partir de la elaboración del primer plan, la salud mental se ha incorporado paulatinamente a las prioridades de salud pública de Chile. Entre otras medidas, la entidad de seguro público (FONASA) ha definido nuevas actividades de salud mental con respaldo financiero; han aumentado los fondos protegidos en el presupuesto de salud, tanto para la compra de medicamentos como para la aplicación de programas específicos de salud mental; las siete prioridades de salud mental han pasado a formar parte de los objetivos sanitarios nacionales para la década 2000-2010; se incorporaron las tres prioridades programáticas de salud mental a las enfermedades que cuentan con garantías de atención en la nueva reforma de salud de Chile; y las acciones de salud mental se incluyeron en la cuenta pública que el Ministerio de Salud entrega cada año al país.

\section{DISCUSIÓN}

Los avances logrados en Chile en los últimos 10 años, como el desarrollo de la red de centros de salud mental y la puesta en marcha de programas en diferentes niveles del sector público de salud, son el resultado de la aplicación de un nuevo modelo de atención comunitario en salud mental y psiquiatría. El Plan Nacional ha sido un elemento fun- 
FIGURA 6. Calidad de la vida de los usuarios de los hogares protegidos y servicios de larga estadía de hospitales psiquiátricos, según la escala de la Organización Mundial de la Salud para medir la calidad de la vidaa. Regiones Metropolitana y Quinta de Chile, 1999

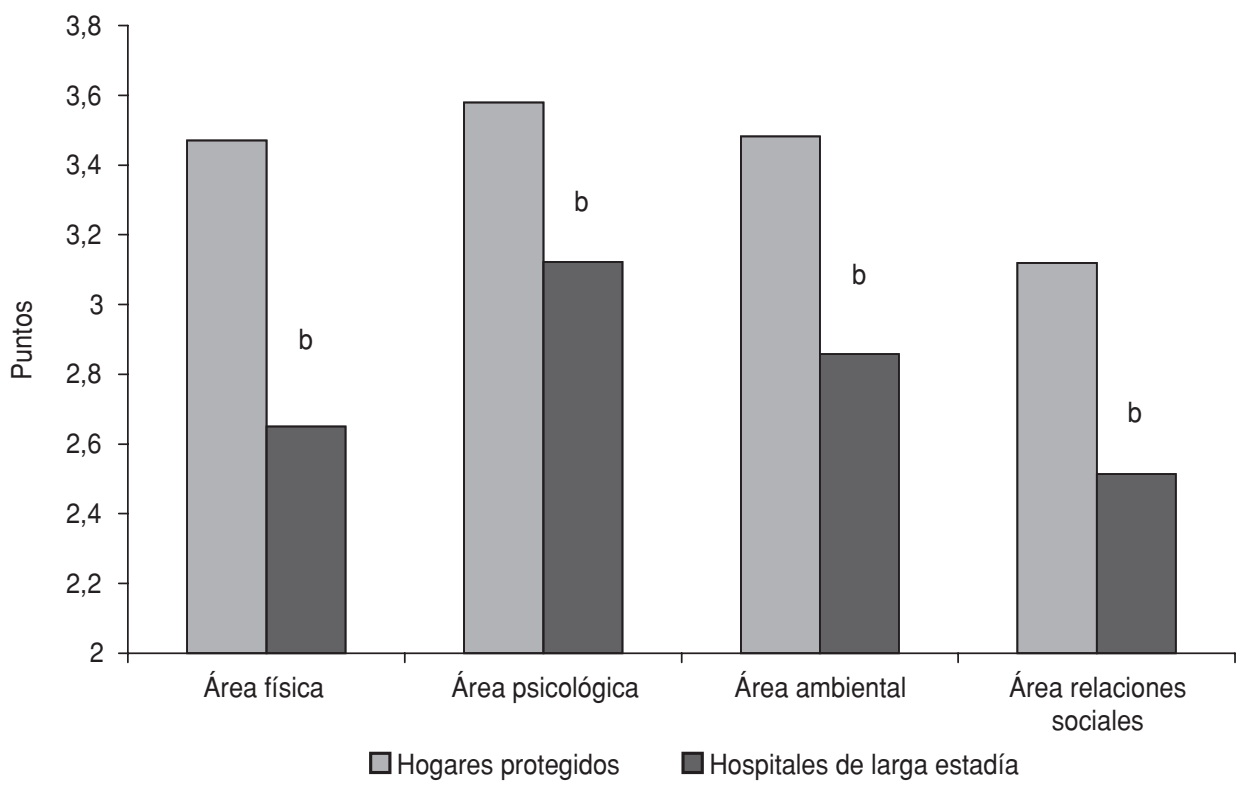

Fuente: Unidad de Salud Mental, Ministerio de Salud, Chile.

a Según datos del Ministerio de Salud de Chile (35) y la versión española del cuestionario de la Organización Mundial de la Salud para evaluar la calidad de la vida (WHOQOL) (38).

b $P>0,005$.

damental en este proceso, ya que ha permitido a los especialistas en salud mental demostrar a los salubristas y a las autoridades sanitarias cómo se puede responder a las necesidades de salud mental de la población con acciones basadas en datos probatorios y que se pueden generalizar a todo el país. El Plan Nacional de Salud Mental y Psiquiatría promovió la inversión en nuevos recursos y la reconversión de los recursos de los servicios tradicionales de psiquiatría. Además, apoyó la gestión de las redes locales de salud mental mediante la formulación de objetivos claros y la elaboración de medidas eficaces. Al mismo tiempo, ha favorecido que las innovaciones incorporadas se adecuen a las realidades socioculturales y a los recursos de cada región del país.

El nuevo modelo de atención de salud mental y psiquiatría se ha insertado en el sector público de salud chileno gracias a que ha pasado a formar parte de los objetivos sanitarios nacionales y de las metas del proceso de reforma del sector de la salud. A esto han contribuido también la aplicación de mecanismos financieros adecuados, la puesta en marcha de un sistema de información específico para la salud mental, el aporte de los programas de salud mental de los 28 servicios de salud del país y la práctica diaria de cientos de equipos multidisciplinarios.
No obstante, la consolidación de este modelo y su continuo desarrollo futuro aún están amenazados por la falta de respaldo legal específico y por la falta de recursos. Tomando en cuenta la fragilidad de muchos procesos sociales y sanitarios en América Latina y la experiencia fructífera de algunos países latinoamericanos que cuentan con leyes de salud mental, se recomienda redoblar los esfuerzos para lograr la promulgación en Chile de leyes que respalden las nuevas redes de salud mental y la plena inserción social de las personas con enfermedades mentales.

Uno de los mayores logros observados durante la puesta en marcha del Plan Nacional de Salud Mental y Psiquiatría fue la contribución de miles de usuarios, familiares, técnicos y profesionales a lo largo de todo el país. Con su motivación, dedicación y creatividad fueron capaces de encontrar caminos concretos para hacer funcionar los nuevos programas y redes y alimentaron con sus experiencias las estrategias nacionales, tanto de gestión como de atención sanitaria. Esta enorme contribución se ha visto limitada, sin embargo, por la insuficiente formación en materia de salud mental y psiquiatría de los egresados de las universidades e institutos profesionales, particularmente en relación con el modelo comunitario. Esto provoca, por 
lo general, que los técnicos y profesionales que se incorporan al sector público de salud, ya sea después de graduarse o porque desean seguir el modelo comunitario de atención sanitaria, deban adquirir las habilidades necesarias sobre la marcha del trabajo y no cuenten con la capacidad de respuesta necesaria para satisfacer las necesidades de los usuarios. En este sentido, una de las debilidades principales del Plan Nacional de Salud Mental y Psiquiatría es no haber previsto la creación de una estructura dedicada al desarrollo de recursos humanos que contemple tanto la formación profesional especializada como la oferta de incentivos por trabajar con el nuevo modelo de atención.

El análisis de los indicadores demuestra que los beneficiarios del sector público de salud en Chile tienen ahora más acceso a los servicios de salud mental que antes del establecimiento del nuevo modelo de atención comunitaria, han ganado en cobertura sanitaria y reciben servicios de mayor calidad. Estas mejoras se caracterizan además por su carácter integral, tanto en los servicios de atención primaria como en los especializados. El nuevo modelo de atención utiliza intervenciones farmacológicas y psicosociales con enfoques preventivos, curativos y de rehabilitación, basados en el trabajo integrado de equipos multidisciplinarios. Otro resultado importante ha sido la cooperación establecida entre el sector de la salud y otros sectores de la sociedad.

Si bien los indicadores presentados están aún lejos de los niveles alcanzados en países desarrollados y de los que espera la población chilena, los resultados obtenidos demuestran que las medidas tomadas han ayudado a reducir significativamente los síntomas y a elevar el grado de satisfacción, la calidad de la vida y las habilidades sociales de los usuarios.
Agradecimientos. Los autores reconocen el apoyo brindado al Plan Nacional de Salud Mental y Psiquiatría por los trabajadores de la Unidad de Salud Mental del Ministerio de Salud, los encargados de salud mental de los 28 servicios de salud del país, los profesionales y técnicos que trabajan en las distintas redes de atención de salud del país, las agrupaciones de usuarios y familiares y, muy especialmente, por las personas con problemas de salud mental.

\section{SYNOPSIS}

\section{The national mental health plan in Chile: 10 years of experience}

Dealing with mental health problems is undoubtedly an increasingly important public health responsibility around the world. In Chile, because of the changes in the epidemiological profile of the population, the lifetime prevalence of mental and behavioral disorders has reached 36\%. In response, the Ministry of Health of Chile, through its Mental Health Unit, prepared the National Plan for Mental Health and Psychiatry. The Plan establishes objectives, strategies, and steps to improve the well-being and mental health of Chileans. This piece describes the model of care for mental health and psychiatry used in Chile's public health care system, analyzes the main difficulties encountered and the achievements made in the 10 years that the Plan has been in place, and makes recommendations for improving the Plan. Over the 10-year period, the new model for mental health and psychiatry has managed to make a place for itself in the public health care system. Indicators show that the beneficiaries of the public health care system in Chile now have greater access to mental health services than before the new model of community care was established, have broader health care coverage, and receive better quality services.

Key words: National health programs, health planning, health plan implementation, mental health, Chile. 


\section{REFERENCIAS}

1. Organización Mundial de la Salud. Informe sobre la salud en el mundo, 2001. Salud mental: nuevos conocimientos, nuevas esperanzas. Ginebra: OMS; 2001.

2. Organización Mundial de la Salud. Invertir en salud mental. Ginebra: OMS; 2004.

3. Concha M, Aguilera X, Gonzáles C, Rodríguez L, Bedregal P, Muñoz Y, et al. Estudio de costo-efectividad de intervenciones para los principales problemas de salud pública. Santiago: Ministerio de Salud; 1993.

4. Vicente B, Rioseco P, Saldivia S, Kohn R, Torres S. Estudio chileno de prevalencia de patología psiquiátrica (SSM-IIIR/ CIDI) (ECCP). Rev Med Chile. 2002;130: 527-36.

5. Minoletti A, López C. Las enfermedades mentales en Chile: magnitud y consecuencias. Santiago: Ministerio de Salud; 1999.

6. Araya R. Trastornos mentales en la práctica médica general. Santiago: Laboratorio Saval; 1997.

7. Minoletti A, Figueroa D, Monrreal V, Pemjeam A. Costo económico del abuso y dependencia del alcohol en Chile. Santiago: Ministerio de Salud; 1999.

8. Barrientos M, Aguilera X, Salas J. La carga de enfermedad en Chile. Santiago: Ministerio de Salud; 1996.

9. República de Chile, Ministerio de Salud. Políticas y Plan Nacional de Salud Mental. Santiago: Ministerio de Salud; 1993.

10. República de Chile, Ministerio de Salud. Plan Nacional de Salud Mental y Psiquiatría. Santiago: Ministerio de Salud; 2000.

11. Alarcón RD, Aguilar-Gaxiola SA. Mental health policy developments in Latin America. Bull World Health Organ. 2000;78(4):483-90.

12. Ministerio de Planificación y Cooperación. Impacto de las políticas públicas en la situación de salud, 1990-1996. Santiago: Ministerio de Planificación y Cooperación; 1998. Hallado en: http:/ / www.mideplan.cl/admin/ docdescargas/centrodoc/centrodoc 60 . pdf. Acceso el 14 de agosto de 2005.
13. Sandoval H. Mejor salud para los chilenos: fundamentos sanitarios, políticos y financieros de la necesidad y oportunidad de hacer una reforma al sistema de salud chileno. Cuad Med Soc. 2004; 43(1):5-20.

14. República de Chile, Ministerio de Planificación y Cooperación. Encuesta de Caracterización Socioeconómica Nacional 2003. Santiago: Ministerio de Planificación y Cooperación; 2004. Hallado en: http:/ / www.mideplan.cl/publico/ publicaciones. . php? secid $=4 \&$ catid $=76$. Acceso el 17 de julio de 2005.

15. República de Chile, Ministerio de Planificación y Cooperación. Situación de salud 2000. Informe ejecutivo. Santiago: Ministerio de Planificación y Cooperación; 2001. Hallado en: http:/ / epi.minsal. $\mathrm{cl} /$ epi/html/sdesalud/sdsalud1.htm Acceso el 17 de julio de 2005.

16. Sepúlveda A. El significado histórico de la reforma de la atención de salud en Chile. Cuad Med Soc. 2004;43(1):21-36.

17. World Health Organization. Core health indicators 2003. Geneva: WHO; 2004

18. Ipinza M. Tendencias recientes en salud y atención de salud en Chile. Cuad Med Soc. 2004;44(1):47-52.

19. Fondo de las Naciones Unidas para la Infancia. Estado mundial de la infancia, 2001. Primera infancia. Nueva York: UNICEF; 2001.

20. World Bank. The health of adults in the developing world. Washington, D.C.: World Bank; 1992.

21. Chile, Ministerio de Salud. Situación de salud de Chile 2000. Santiago: Ministerio de Salud; 2002.

22. Pemjeam A. El proceso de reestructuración de la atención en salud mental y psiquiatría en Chile (1990-1996). Cuad Med Soc. 1996;37(2):5-17.

23. World Health Organization. Mental health policy, plans and programmes. Geneva: WHO; 2003.

24. World Health Organization. Organization of services for mental health. Geneva: WHO; 2003.

25. World Health Organization. Planning and budgeting to deliver services for mental health. Geneva: WHO; 2003.
26. Chile, Ministerio de Salud. Norma técnica: organización de una red de servicios de salud mental y psiquiatría y orientaciones para su aplicación. Santiago: Ministerio de Salud; 1999.

27. World Health Organization. Mental health financing. Geneva: WHO; 2003.

28. World Health Organization. Quality improvement for mental health. (Mental health policy and service guidance package). Geneva: WHO; 2003.

29. Monrreal V, Montoya C. Desigualdades en salud mental. Chile 1997-2001. Cuad Med Soc. 2004;44(2):91-101.

30. World Health Organization. Advocacy for mental health. (Mental health policy and service guidance package). Geneva: WHO; 2003.

31. World Health Organization. Mental health legislation and human rights. (Mental health policy and service guidance package). Geneva: WHO; 2003.

32. Chile, Ministerio de Salud. Los objetivos sanitarios para la década 2000-2010. 1 . $^{\text {a }}$ ed. Santiago: Ministerio de Salud; 2002.

33. Universidad de Chile, Facultad de Medicina, Escuela de Salud Pública. Evaluación de la efectividad del Programa para la Detección, Diagnóstico y Tratamiento Integral de la Depresión en Atención Primaria. Informe final. Santiago: Universidad de Chile; 2002.

34. Saldivia S, Vicente B, Kohn R, Rioseco P, Torres $\mathrm{S}$. Use of mental health services in Chile. Psych Serv. 2004;55:71-6.

35. Chile, Ministerio de Salud. Estudio de hogares protegidos. Santiago: Ministerio de Salud; 2000.

36. Chile, Ministerio de Salud. Estructura, procesos, flujos, clasificación de unidades de atención ambulatoria e impacto del modelo comunitario en salud mental y psiquiatría en población usuaria diagnosticada con esquizofrenia, en el sistema público de salud, 2002. Santiago: Ministerio de Salud; 2002.

37. Ministerio de Salud. Evaluación del programa de hogares protegidos para pacientes con esquizofrenia. Santiago: Ministerio de Salud; 2004.

38. Lucas R. Versión española del WHOQOL. Madrid: Ediciones Ergon; 1998.

\section{Resolve to be yourself; and know that he who finds himself, loses his misery.}

English poet and critic (1822-1888) 